\title{
RESEARCH
}

Open Access

\section{Clinical-MRI radiomics enables the prediction of preoperative cerebral spinal fluid dissemination in children with medulloblastoma}

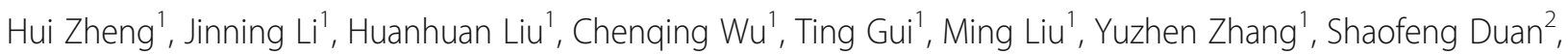
Yuhua $\mathrm{Li}^{1 *}$ and Dengbin Wang ${ }^{1 *}$ (i)

\begin{abstract}
Background: Medulloblastoma (MB) is the most common pediatric embryonal tumor. Accurate identification of cerebral spinal fluid (CSF) dissemination is important in prognosis prediction. Both MRI of the central nervous system (CNS) and CSF cytology will appear false positive and negative. Our objective was to investigate the added value of preoperative-enhanced T1-weighted image-based radiomic features to clinical characteristics in predicting preoperative CSF dissemination for children with MB.

Materials and methods: This retrospective study included 84 children with histopathologically confirmed MB between November 2006 and November 2018 (training cohort, $n=60$; internal validation cohort, $n=24$ ). A set of cases between December 2018 and February 2020 were used for external validation $(n=40)$. The children with normal head and spine magnetic resonance images (MRI) and no subsequent dissemination in 1 year were diagnosed as non-CSF dissemination. The CSF dissemination was manifested as intracranial or intraspinal nodularenhanced lesions. Clinical features were collected, and conventional MRI features of preoperative head MRI examinations were evaluated. A total of 385 radiomic features were extracted from preoperative-enhanced T1weighted images. Minimum redundancy, maximum correlation, and least absolute shrinkage and selection operator were performed to select the features with the best performance in predicting preoperative CSF dissemination. A combined clinical-MRI radiomic prediction model was developed using multivariable logistic regression. Receiver operating curve analysis (ROC) was used to validate the predictive performance. Nomogram and decision curve analysis (DCA) were developed to evaluate the clinical utility of the combined model.

Results: One clinical and nine radiomic features were selected for predicting preoperative CSF dissemination. The combined model incorporating clinical and radiomic features had the best predictive performance in the training cohort with an AUC of 0.89. This was validated in the internal and external cohorts with AUCs of 0.87 and 0.73 . The clinical utility of the model was confirmed by a clinical-MRI radiomic nomogram and DCA.

(Continued on next page)
\end{abstract}

\footnotetext{
*Correspondence: liyuhua@xinhuamed.com.cn;

wangdengbin@xinhuamed.com.cn

'Department of Radiology, Xinhua Hospital affiliated to Shanghai Jiao Tong

University School of Medicine, Shanghai, China

Full list of author information is available at the end of the article
}

C C The Author(s). 2021 Open Access This article is licensed under a Creative Commons Attribution 4.0 International License, which permits use, sharing, adaptation, distribution and reproduction in any medium or format, as long as you give appropriate credit to the original author(s) and the source, provide a link to the Creative Commons licence, and indicate if changes were made. The images or other third party material in this article are included in the article's Creative Commons licence, unless indicated otherwise in a credit line to the material. If material is not included in the article's Creative Commons licence and your intended use is not permitted by statutory regulation or exceeds the permitted use, you will need to obtain permission directly from the copyright holder. To view a copy of this licence, visit http://creativecommons.org/licenses/by/4.0/ The Creative Commons Public Domain Dedication waiver (http://creativecommons.org/publicdomain/zero/1.0/) applies to the data made available in this article, unless otherwise stated in a credit line to the data. 
(Continued from previous page)

Conclusions: The combined model incorporating clinical, conventional MRI, and radiomic features could be applied to predict preoperative CSF dissemination for children with MB as a noninvasive biomarker, which could aid in risk evaluation.

Keywords: Children, Cerebral spinal fluid dissemination, Magnetic resonance imaging, Medulloblastoma, Radiomics

\section{Background}

Medulloblastoma (MB) is the most common embryonal tumor located in the posterior cranial fossa. It usually affects young children before the age of 9 years [1]. Approximately $30 \%$ of children with $\mathrm{MB}$ have cerebral spinal fluid (CSF) dissemination with either magnetic resonance imaging (MRI) suggestive of disseminative nodules or CSF cytology demonstrated tumor cells [2].

At present, the standard treatment for $\mathrm{MB}$ is maximum resection followed by risk-adapted adjuvant chemoradiotherapy. Depending on the absence or presence of adverse prognostic factors including age younger than 3 years, anaplastic histopathologic subtype, CSF dissemination, and residual tumor greater than $1.5 \mathrm{~cm}$ in diameter, the child with $\mathrm{MB}$ will be stratified into an average or high-risk group [3]. The 2016 World Health Organization classification of tumors of the central nervous system (CNS) grades MB by its molecular profiling, which has a more reliable performance in stratifying the risk of $\mathrm{MB}$ and guiding clinical treatment strategies [4]. Although the advances regarding the molecular characteristics of $\mathrm{MB}$ could aid risk stratification, accurate identification of CSF dissemination remains important in prognosis prediction.

Both MRI of the CNS and CSF cytology will appear false positive and negative. On the one hand, MRI of the CNS is not able to detect early dissemination, while on the other hand, the equivocal findings such as enhanced meningeal thickening and nerve roots clumping may be misdiagnosed as dissemination $[5,6]$. Because of technical or sampling problems, CSF cytology may fail to detect the tumor cells. Multiple CSF sampling may improve the diagnostic accuracy, but at the cost of additional discomfort to children as more lumbar punctures would be required [7]. Therefore, establishing a noninvasive biomarker for predicting CSF dissemination will be of great significance.

MRI is the modality of choice for risk stratification in children with MB. It can provide more information than conventional MRI features evaluated by radiologists, because images are not data mined [8]. To the best of our knowledge, there is no published data concerning the radiomics predicting the metastasis risk of $\mathrm{MB}$ in children.

Radiomics is an approach that is able to extract highthroughput quantitative features from medical images
[9]. In theory, radiomic features are able to reflect biological characteristics of the tumor and could aide in differential diagnosis, and prognosis and distant metastasis prediction, among others [10-12]. In this present study, we investigated the added value of enhanced T1weighted image-based radiomics to clinical characteristics in predicting preoperative CSF dissemination for children with MB.

\section{Materials and methods \\ Patients}

This retrospective study was approved by the Institutional Ethics Committee of Xinhua Hospital Affiliated to Shanghai Jiao Tong University School of Medicine (ethics approval number: XHEC-D-2020-136), and therefore, informed consent was waived.

The inclusion criteria included (i) the availability of preoperative head MRI with diagnostic quality, (ii) availability of spine MRI with diagnostic-quality performed pre- or postoperative but before adjuvant therapy, (iii) children without CSF dissemination confirmed by head and spine MRI required follow-up results for 1 year, and (iv) without any previous treatment. The exclusion criteria included (i) insufficient head and spine MRI quality, (ii) without 1-year follow-up results for children without CSF dissemination, (iii) previous treatment, and (iv) equivocal findings on spine MRI such as enhanced meningeal thickening and nerve roots clumping.

Children with pathologically confirmed MB were reviewed between November 2006 and November 2018 . The children met the including and excluding criteria were randomly distributed to the training and internal validation cohorts according to a 7:3 ratio. A set of cases between December 2018 and February 2020 were used for external validation of the prediction model.

Clinical features, including age, gender, and histopathologic subtype (classic, desmoplastic-nodular, MB with extensive nodularity, and large cell/anaplastic) of all children with $\mathrm{MB}$, were collected via medical records.

The workflow of this retrospective study is displayed in Fig. 1.

\section{Imaging acquisition}

All of the children underwent pre- and postoperative contrast-enhanced T1-weighted head MRI using a 3.0-T MRI scanner (Signal HDxt, GE Healthcare, Boston, MA, 


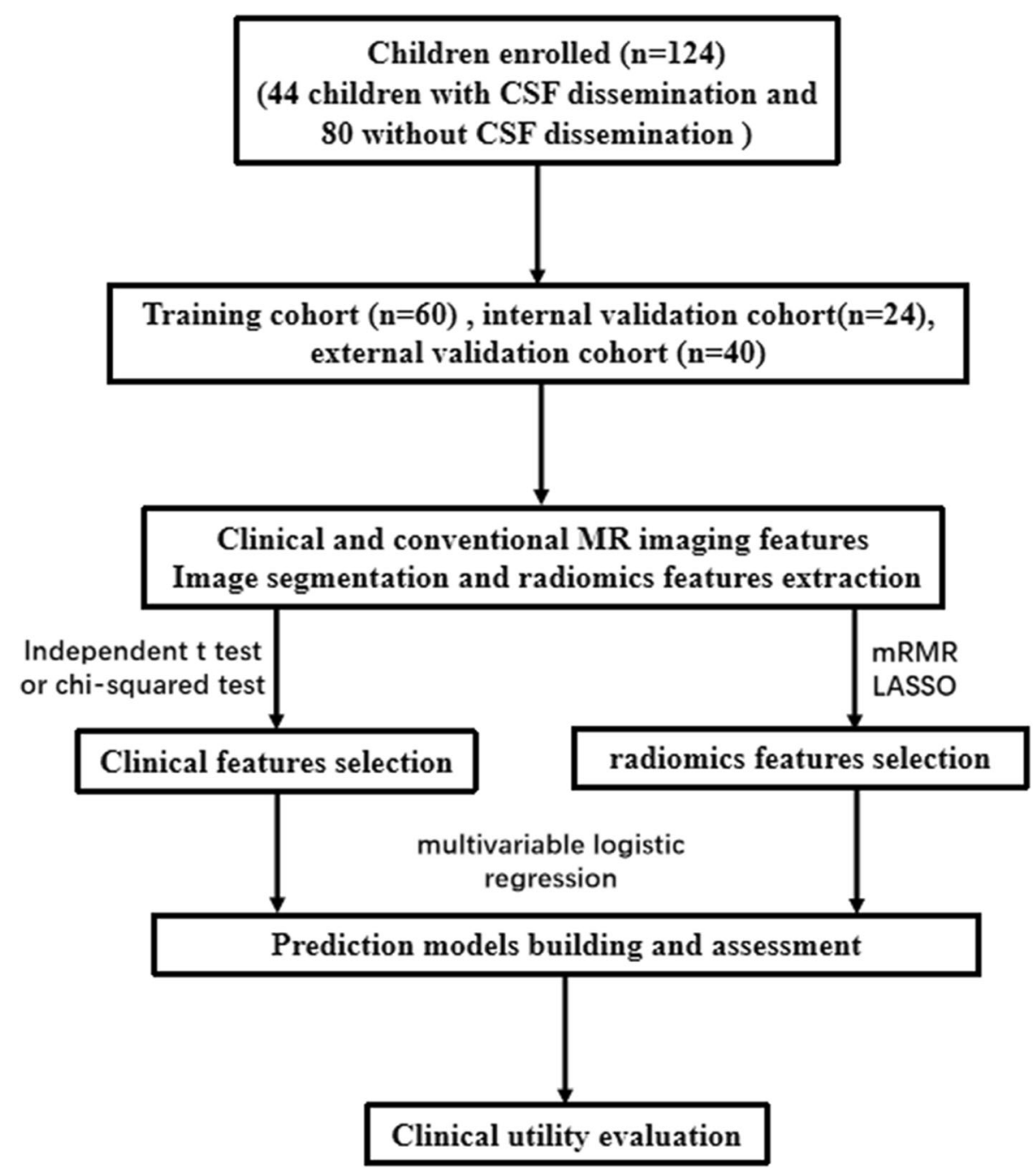

Fig. 1 Workflow of this study. CSF cerebral spinal fluid; MRI magnetic resonance imaging; mRMR minimum redundancy and maximum correlation; LASSO least absolute shrinkage and selection operator

USA) with an 8-channel head coil. The contrast-enhanced T1weighted images were obtained with a slice thickness of $4 \mathrm{~mm}$ and a matrix of $512 \times 512$. Children also underwent contrastenhanced spine MRI, which was given either preoperative or postoperative but before adjuvant therapy. Axial and sagittalenhanced T1-weighted images were obtained with a thickness of $3 \mathrm{~mm}$ and gap of $0 \mathrm{~mm}$. The detailed parameters for head and spine MRI are provided in Appendix E1 and E2.

The contrast material was administrated with a dose of 0.1 $\mathrm{mmol} / \mathrm{kg}$ (Gadopentetate Dimeglumine, Beilu, Beijing, China). Children that were unable to remain motionless during the MRI examination were sedated with chloral hydrate $(0.5 \mathrm{mg} / \mathrm{kg})$.

\section{Qualitative image evaluation}

\section{Conventional MR imaging evaluation}

All of the preoperative head MR images were reviewed by two pediatric radiologists (H.Z and J.N.L, with 9 and
12 years of experience, respectively, in pediatric neuroradiology). Discrepancies were resolved by consulting with a third pediatric neuroradiologist (Y.H.L) with 30 years of experience. A set of conventional MRI features were evaluated, including the location of the tumor, contrast enhancement pattern, intratumoral necrosis, hemorrhage, calcification, peritumoral edema, and the minimal apparent diffusion coefficient (minADC) value. A description for these features is provided in Appendix E3.

\section{CSF dissemination assessment}

The enhanced T1-weighted peri-surgical head and spine MR images were used for CSF dissemination assessment. Children with normal head and spine MR images and no subsequent dissemination at the 1-year follow-up were regarded as the non-CSF dissemination group. CSF dissemination was manifested as intracranial or 
intraspinal nodular lesions and leptomeningeal enhancement. Children with equivocal findings on head and spine MR images were excluded.

\section{Image segmentation and radiomic feature extraction}

The delineation of $\mathrm{MB}$ and radiomic extraction is shown in Fig. 2. One radiologist (H.Z with 9 years of experience in pediatric neuroradiology) determined the volume of interest (VOI) of the tumor on preoperative contrastenhanced T1-weighted images using software package ITK-SNAP (www.itksnap.org). The peritumoral edema and surrounding vessels were carefully avoided. The segmentations were examined by another pediatric neuroradiologist (M.L with 20 years of experience). Both were blinded to the CSF dissemination status.

By using an in-house software Analysis Kit (GE healthcare), a total of 385 radiomic features were extracted automatically from the VOI including histogram parameters, volume and shape parameters, Haralick features, gray-level co-occurrence parameters, and gray-level runlength matrix parameters.

\section{Feature selection, prediction model building, and}

\section{validation}

Differences in clinical and conventional MR imaging characteristics between the children with and without CSF dissemination in the training, internal, and external validation cohorts were assessed. A clinical prediction model was built using the features with significant differences.

In the training cohort, minimum redundancy and maximum correlation (mRMR) and least absolute shrinkage and selection operator (LASSO) were performed to reduce dimension and select the radiomic features with the strongest CSF dissemination-related correlation. The radiomic score (rad-score) of each child with $\mathrm{MB}$ was calculated by adding all of the products of the selected features and their corresponding coefficients, after which a radiomic prediction model was built. A combined clinical-radiomic prediction model was developed using multivariable logistic regression, which incorporated selected clinical features and radscores. A clinical-radiomic nomogram was then constructed in the training cohort.

The receiver operating characteristic (ROC) curves were used to evaluate the predictive performance of the clinical, radiomic, and combined models in both the training and two validation cohorts. The area under the curves (AUC), sensitivities, specificities, and accuracy were calculated. And then, the Hosmer-Lemeshow test was performed to assess the goodness-of-fit of the combined model. Decision curve analysis (DCA) was implemented to determine the clinical usefulness of the prediction models in the training cohort at different threshold probability.

\section{Statistical analysis}

The differences of clinical and conventional MRI characteristics between the training and two validation cohorts, as well as between children with and without CSF dissemination in their respective cohorts were evaluated using independent $t$ tests or chi-squared tests according to the type of the data. The optimal values of the ROCs were determined using Youden's index. Delong's test was used to assess the differences in the AUC values

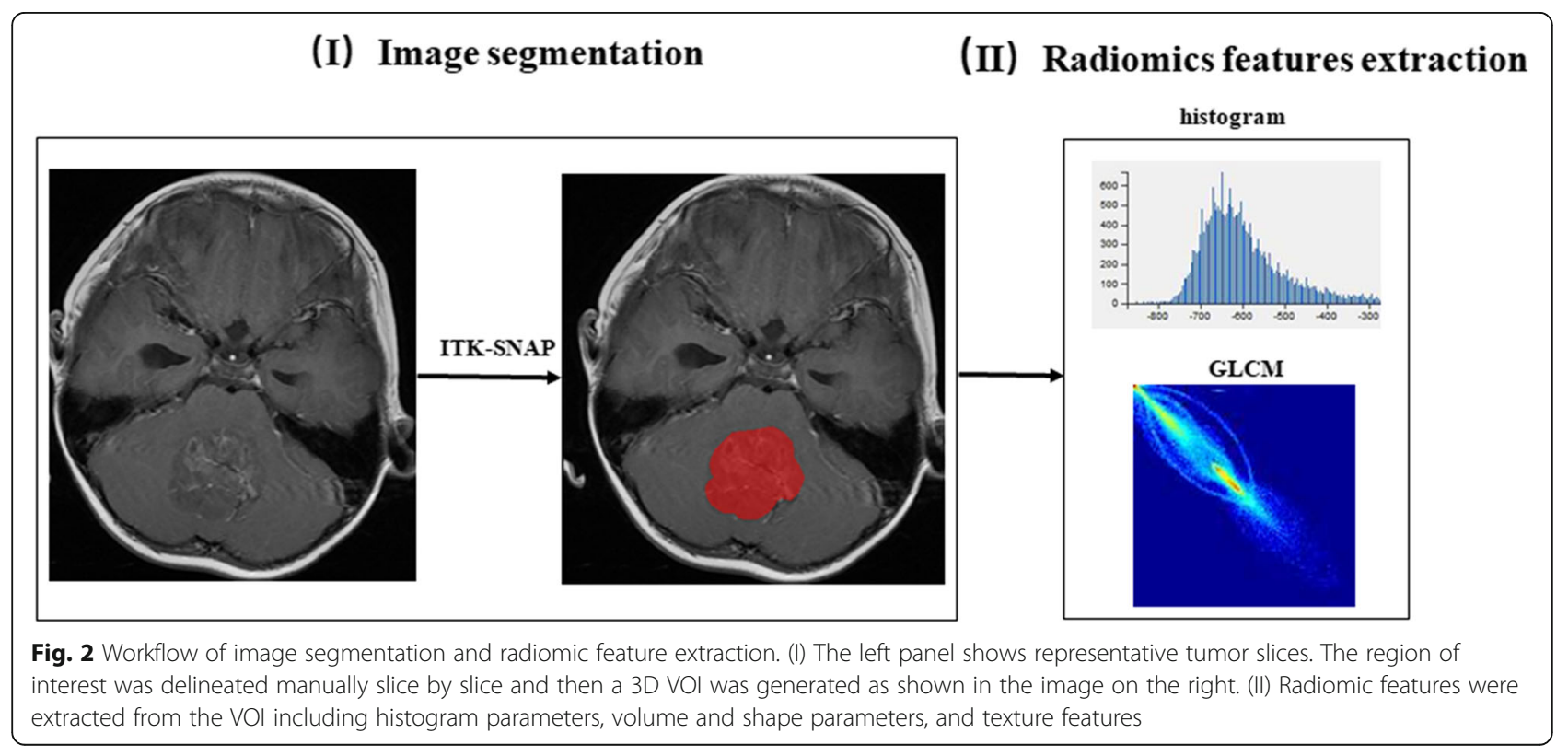


between the clinical and clinical-radiomic combined models.

All statistical analyses were performed using the $\mathrm{R}$ software package (version 3.4.2, http://www.Rproject. org). The ROC curves were performed using the "pROC" package. Multivariate logistic regression was plotted with the "rms" package. The Hosmer-Lemeshow test was conducted using the "Resource Selection" package. DCA was developed with the function of "dca.R". $P<0.05$ was set as statistical significance.

\section{Results}

\section{Clinical features of the children}

According to the inclusion criteria, a total of 124 children with $M B$ were recruited in this study (60 children in the training cohort, 24 and 40 children in the internal and external validation cohorts, respectively). None of the children experience extra-CNS metastases. Fortyfour children (35.6\%) were confirmed to have CSF dissemination by head and spine MRI evaluation. Five children had only intracranial dissemination while 39 children had both intracranial and intraspinal dissemination. Eighty children without CSF dissemination were identified by MRI evaluation and follow-up information.

The clinical features of the children in the training and two validation cohorts were displayed in Table 1 . There were no significant differences among the three cohorts in terms of clinical features except for histopathological subtype. In the training cohort, children were significantly younger $(P=0.028)$ in the CSF dissemination group than in the non-CSF dissemination group.

\section{Feature selection, prediction model building, and validation}

In the training cohort, nine radiomic features with the strongest CSF dissemination features were selected using mRMR and LASSO shown in Fig. 3. The rad-score was calculated using the following formula:

Table 1 Clinical and conventional MRI characteristics of children with MB

\begin{tabular}{|c|c|c|c|c|c|c|c|c|c|}
\hline \multirow[t]{2}{*}{ Characteristics } & \multicolumn{2}{|c|}{ Training cohort $(n=60)$} & \multirow[t]{2}{*}{$\begin{array}{l}p \\
\text { value }\end{array}$} & \multicolumn{2}{|c|}{$\begin{array}{l}\text { Internal validation cohort }(n= \\
\text { 24) }\end{array}$} & \multirow[t]{2}{*}{$\begin{array}{l}p \\
\text { value }\end{array}$} & \multicolumn{2}{|c|}{$\begin{array}{l}\text { External validation cohort }(n= \\
40)\end{array}$} & \multirow[t]{2}{*}{$\begin{array}{l}p \\
\text { value }\end{array}$} \\
\hline & $\begin{array}{l}\text { Non-metastasis } \\
(n=38, \%)\end{array}$ & $\begin{array}{l}\text { Metastasis }(n \\
=22, \%)\end{array}$ & & $\begin{array}{l}\text { Non-metastasis } \\
(n=15, \%)\end{array}$ & $\begin{array}{l}\text { Metastasis } \\
(n=9, \%)\end{array}$ & & $\begin{array}{l}\text { Non-metastasis } \\
(n=27, \%)\end{array}$ & $\begin{array}{l}\text { Metastasis } \\
(n=13, \%)\end{array}$ & \\
\hline $\begin{array}{l}\text { Age (mean } \pm S D \text {, } \\
\text { years) }\end{array}$ & $6.2 \pm 3.5$ & $4.3 \pm 2.5-$ & 0.028 & $5.1 \pm 2.9$ & $3.6 \pm 1.7$ & 0.154 & $6.3 \pm 3.4$ & $6.4 \pm 3.3$ & 0.937 \\
\hline Gender (\%) & & & 1.000 & & & 0.597 & & & 0.360 \\
\hline Male & $26(68.4)$ & $15(68.2)$ & & $7(46.7)$ & $6(66.7)$ & & $13(48.1)$ & $9(69.2)$ & \\
\hline Female & 12 (31.6) & $7(31.8)$ & & $8(53.3)$ & $3(33.3)$ & & $14(51.9)$ & $4(30.8)$ & \\
\hline $\begin{array}{l}\text { Histopathologic } \\
\text { subtype (\%) }\end{array}$ & & & 0.632 & & & 0.444 & & & 0.748 \\
\hline Classic & $33(86.8)$ & $17(77.3)$ & & $10(66.7)$ & $8(88.9)$ & & $21(77.8)$ & $11(84.6)$ & \\
\hline $\begin{array}{l}\text { Large cell/ } \\
\text { anaplastic }\end{array}$ & $1(2.6)$ & $1(4.5)$ & & $4(26.7)$ & $1(11.1)$ & & $1(3.7)$ & $0(0)$ & \\
\hline $\begin{array}{l}\text { Desmoplastic- } \\
\text { nodule }\end{array}$ & $4(10.5)$ & $4(18.2)$ & & $1(6.7)$ & $0(0.0)$ & & $5(18.5)$ & $2(15.4)$ & \\
\hline Location (\%) & & & 1.0000 & & & 0.507 & & & 1 \\
\hline Midline & $34(89.5)$ & $20(90.9)$ & & $15(100.0)$ & $9(100.0)$ & & $24(88.9)$ & $11(84.6)$ & \\
\hline Nonmidline & $4(10.5)$ & $2(9.1)$ & & $0(0.0)$ & $0(0.0)$ & & $3(11.1)$ & $2(15.4)$ & \\
\hline $\begin{array}{l}\text { Enhancement } \\
\text { pattern (\%) }\end{array}$ & & & 0.4032 & & & 0.052 & & & 0.399 \\
\hline Diffuse & $23(60.5)$ & $17(77.3)$ & & $8(53.3)$ & $9(100.0)$ & & $17(63.0)$ & $10(76.9)$ & \\
\hline Incomplete & $10(26.3)$ & $3(13.6)$ & & $4(26.7)$ & $0(0.0)$ & & $3(11.1)$ & $2(15.4)$ & \\
\hline Minimal & $5(13.2)$ & $2(9.1)$ & & $3(20.0)$ & $0(0.0)$ & & $7(25.9)$ & $1(7.7)$ & \\
\hline $\begin{array}{l}\text { Necrosis or } \\
\text { cyst(\%) }\end{array}$ & & & 0.8664 & & & 0.729 & & & 0.311 \\
\hline None & $5(13.2)$ & $2(9.1)$ & & $1(6.7)$ & $0(0.0)$ & & $0(0)$ & $1(7.7)$ & \\
\hline Small & $22(57.9)$ & 14 (63.6) & & $8(53.3)$ & $5(55.6)$ & & $22(81.5)$ & $9(69.2)$ & \\
\hline Both & $11(28.9)$ & $6(27.3)$ & & $6(40.0)$ & $4(44.4)$ & & $5(18.5)$ & $3(23.1)$ & \\
\hline $\begin{array}{l}\min A D C(\text { mean } \pm \\
\left.S D) \mathrm{mm}^{2} / \mathrm{s}\right)\end{array}$ & $0.4 \pm 0.1$ & $0.4 \pm 0.1$ & 0.191 & $0.5 \pm 0.1$ & $0.4 \pm 0.1$ & 0.0212 & $0.5 \pm 0.1$ & $0.4 \pm 0.1$ & 0.171 \\
\hline
\end{tabular}




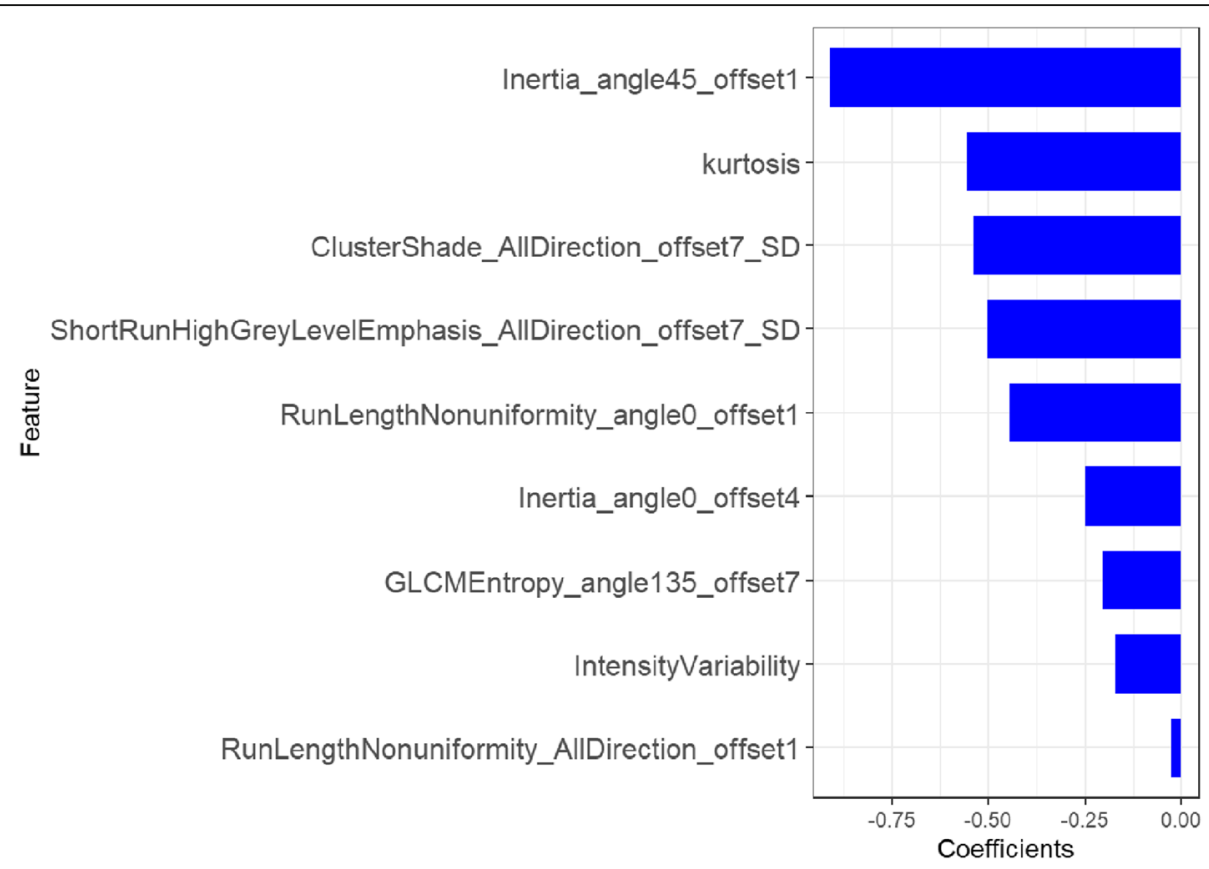

Fig. 3 The selected radiomic features. The role of selected features contributing to the developed rad-score is shown. The selected features are plotted on the $y$-axis, and their regression coefficients in the LASSO analysis are plotted on the $x$-axis

Rad - score $=-0.557 \times$ Kurtosis

$-0.502 \times$ ShortRunHighGreyLevelEmphasis_AllDirection_offset7_SD

$-0.538 \times$ ClusterShade_AllDirection_offset7_SD

$-0.444 \times$ RunLengthNonuniformity_angle $0 \_$offset1

$-0.909 \times$ Inertia_angle 45 _offset 1

$-0.248 \times$ Inertia _ angle 0 _offset 4

$-0.026 \times$ RunLengthNonuniformity _ AllDirection _ offset1

$-0.204 \times$ GLCMEntropy_angle135_offset7

$-0.17 \times$ Intensity/Variability -0.735

The combined clinical-radiomic predictive model was developed using multivariate logistic regression analysis. The AUC of the value of the combined model was 0.89 . And the AUC of the clinical model was much lower (0.67), which indicated that radiomic features could improve performance. The results of the combined predictive model in the training and two validation cohorts were shown in Table 2 (Fig. 4). The nomogram was built with age and rad-score in the training cohort (Fig. 5).

The CSF dissemination predictive performance of combined prediction model was also robust when applied to the internal and external validation cohorts with an AUC of 0.87 and 0.73, respectively. The HosmerLemeshow test demonstrated that the goodness-of-fit of the combined model was high in both the training and two validation cohorts (Fig. 6). DCA revealed that at every threshold probability, it was more beneficial using the combined model than the clinical model alone (Fig. 7).

\section{Discussion}

In the present study with a relatively large case group $(n=124)$, we developed and validated a clinical-radiomic combined model for preoperative prediction of CSF dissemination in children with MB. The nomogram combined the age of the children and a preoperativeenhanced T1-weighted image-based radiomic signature. The results demonstrated that compared with the prediction model built using clinical features alone, the radiomic signature could improve the predictive performance of CSF dissemination. The AUC was increased from 0.67 to 0.89 in the training group. The sensitivity, specificity, NPV, and PPV were relatively higher both in the training and two validation cohorts. The higher sensitivity indicated that the combined model could identify children with CSF dissemination accurately. These children should undergo examinations with a higher specificity, such as CNS, MRI, and CSF cytology to evaluate the dissemination staging.

Children with CNS dissemination should be given aggressive therapy, as prognosis is usually poor, no matter what the molecular subgroup is. It is, therefore, paramount to establish a method to accurately diagnose CNS dissemination. Traditionally, diagnosis of CSF dissemination encompassed comprehensively analyzing the combined results of CNS, MRI, and CSF cytology [7, 13], both of which may over- or underestimate the CSF dissemination. On the one hand, overestimation leads to higher risk stratification and unnecessarily high radiotherapy doses, which could affect the quality of life of 
Table 2 Performance of the combined clinical-radiomic predictive model

\begin{tabular}{llll}
\hline & Training cohort & Internal validation CohortcohorCohort & External validation cohort \\
\hline AUC (95\%) & $0.89(0.81-0.97)$ & $0.87(0.71-1.00)$ & $0.73(0.56-0.85)$ \\
Accuracy & 0.82 & 0.83 & 0.73 \\
Sensitivity & 0.91 & 0.87 & 0.78 \\
Specificity & 0.76 & 0.87 & 0.85 \\
\hline
\end{tabular}

children with MB. On the other hand, underestimation leads to lower risk stratification and lower radiotherapy doses, which could increase the possibility of tumor recurrence and decrease survival. Identification of tumor cells by CSF cytology is an important element of the diagnosis of CSF dissemination. However, a negative result must be viewed with caution due to high rates of technique and sampling errors [14]. Multiple lumber punctures may improve tumor cell detection, but cause increased pain to children and also delays corresponding adjuvant treatment.

In this retrospective study, CSF cytology results were not obtained. Therefore, the diagnosis of CSF dissemination depended on head and spine MR images. Children without CSF dissemination were identified by negative head and spine MRI and outcome at the 1-year followup. This diagnosis strategy is consistent with the literature determining the status of CSF dissemination by using overall survival [6]. The equivocal findings in spine MRI such as linear enhancement, clumping, and enhancement of nerve roots can be misdiagnosed as CSF dissemination, all of which can be identified in preoperative MRI. Therefore, extreme care is needed and close follow-up is required to confirm any initial diagnosis.

Radiomics can evaluate the intratumoral heterogeneity and predict prognosis by extracting high throughput quantified features using medical images. A previous study indicated that when the prediction accuracy of the radiomic signature was constructed by using 24 radiomic features from preoperative computerized tomography images for lymph node metastasis in patients with colorectal cancer, the concordance index was 0.773 [15]. In another study, when the radiomic signature was constructed using T2-weighted images, the prediction accuracy was improved compared with a model built only using clinical features for synchronous distant metastasis in patients with rectal cancer, where the AUC for the combined model and clinical model was 0.827 and 0.779 , respectively [12].

In CNS tumors, radiomics is mainly applied to differential diagnosis of tumors, World Health Organization grading, molecular profiling, and prognosis prediction in the adult population [16-19]. However, for pediatric $\mathrm{MB}$, research is focused on differential diagnosis of tumors in the posterior cranial fossa and in molecular subtype prediction $[20,21]$. Therefore, it is worth exploring an imaging biomarker to detect CSF dissemination based on the MRI radiomic features of pediatric MB.

According to previous reports, the CSF dissemination probability of medulloblastoma has a close relationship to molecular subgroups. For example, the prognosis of children with group 3 is very poor and prone to early metastasis [22]. Some MRI features of MB are reliable to predict molecular subgroups, especially their location and degree of enhancement. Group 3 tumors are mainly located in the midline of posterior cranial fossa with
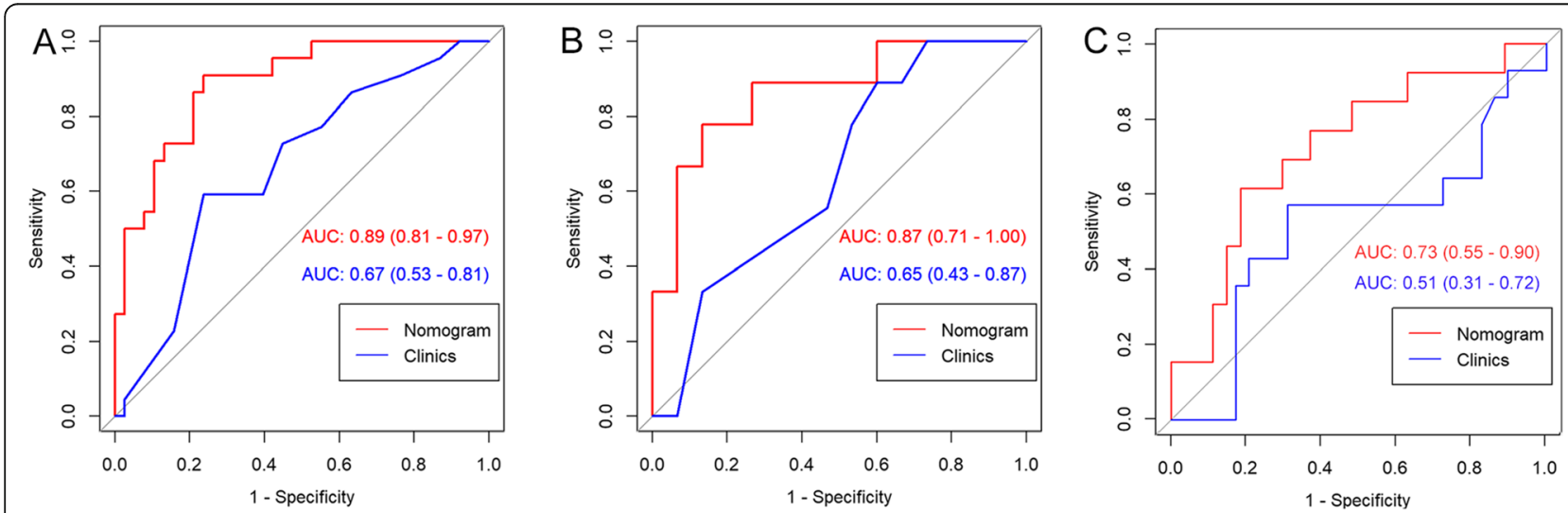

Fig. 4 ROC curves of the traditional feature model and nomogram. a The AUC was significantly different ( 0.67 and 0.89 , respectively) between the clinical model and the nomogram in the training cohort. $\mathbf{b}, \mathbf{c}$ The AUC of the nomogram was significantly higher than a clinical model in the internal and external cohorts. Delong's test showed that the differences between the ROC curves of the nomogram and clinical model were significantly different in both the training and two validation cohorts. ROC receiver operating characteristic, AUC area under the curve 


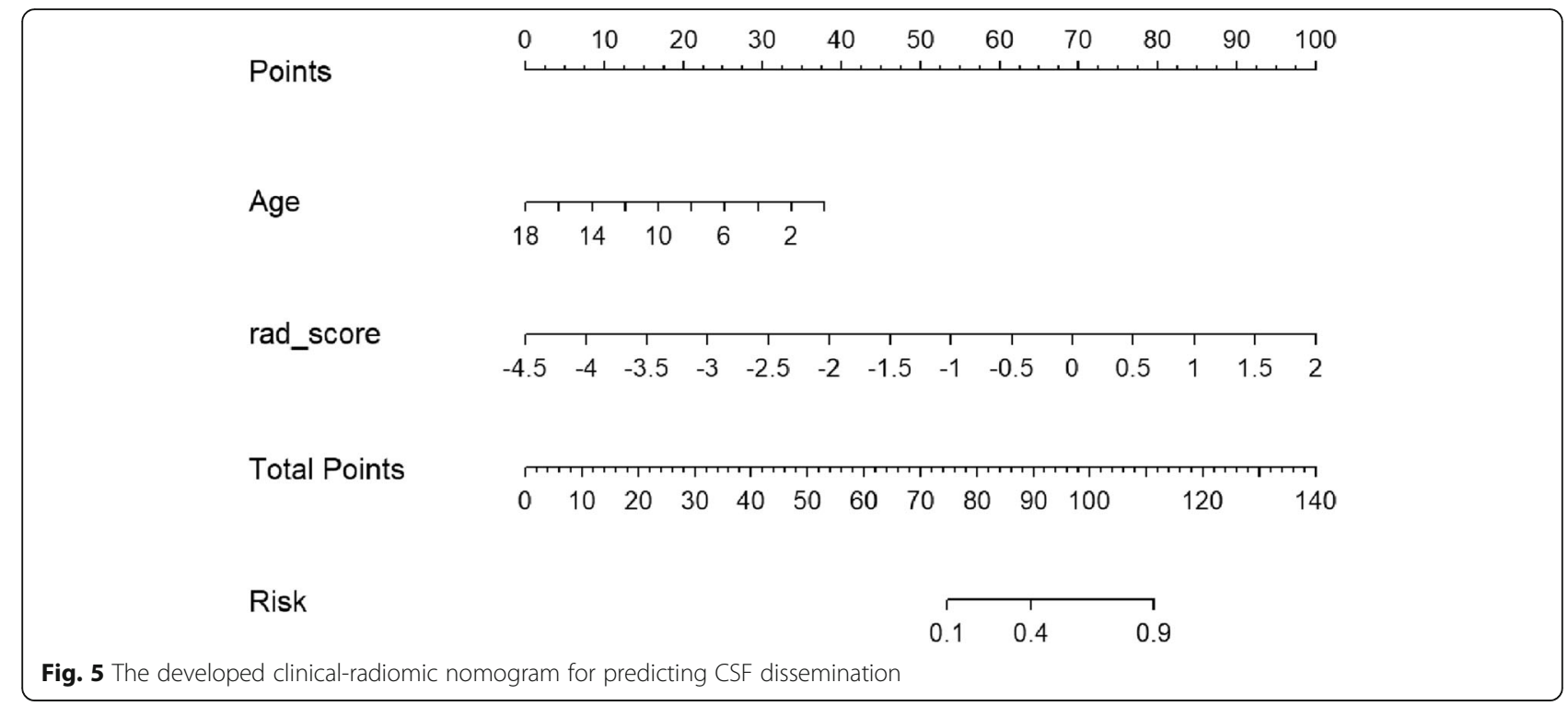

strong enhancement, whereas group 4 tumors, although also located at the midline, show minimal enhancement [23]. These imaging features are able to be assessed on enhanced T1-weighted images, and as such, we chose to use this type of enhancement to extract radiomic features.

In the present study, the results demonstrated the added value of radiomics to clinical and conventional MRI characteristics in predicting CSF dissemination for pediatric MB. The higher sensitivity and accuracy of the combined model compared with the clinical model alone is encouraging. It is likely that the probability of CSF dissemination could be calculated using the proposed combined model nomogram.

The CSF dissemination prediction model included the clinical variable of age, as group 3 tumors are prone to occur in younger children and infants [24]. Regarding to radiomic features, most of the selected features used to determine the rad-score were texture features. This is consistent with the better performance of texture features compared with first-order features in tumor prognosis prediction, as these features may reflect intratumoral heterogeneity [25].

There are several limitations to the current study. First, due to the small sample size of the cohort, useful conventional MRI features for the predictive model could not be used because of selective bias. The association between conventional MRI features and CSF dissemination in children with $\mathrm{MB}$ should be further explored in a large-scale study. Second, due to the retrospective nature of the study, an external validation from another institution was not performed. A multi-center prospective research with different MRI scanning protocols is needed to evaluate the generalization of our predictive model. Third, only enhanced T1-weighted images were used in this radiomic research. Multiparametric
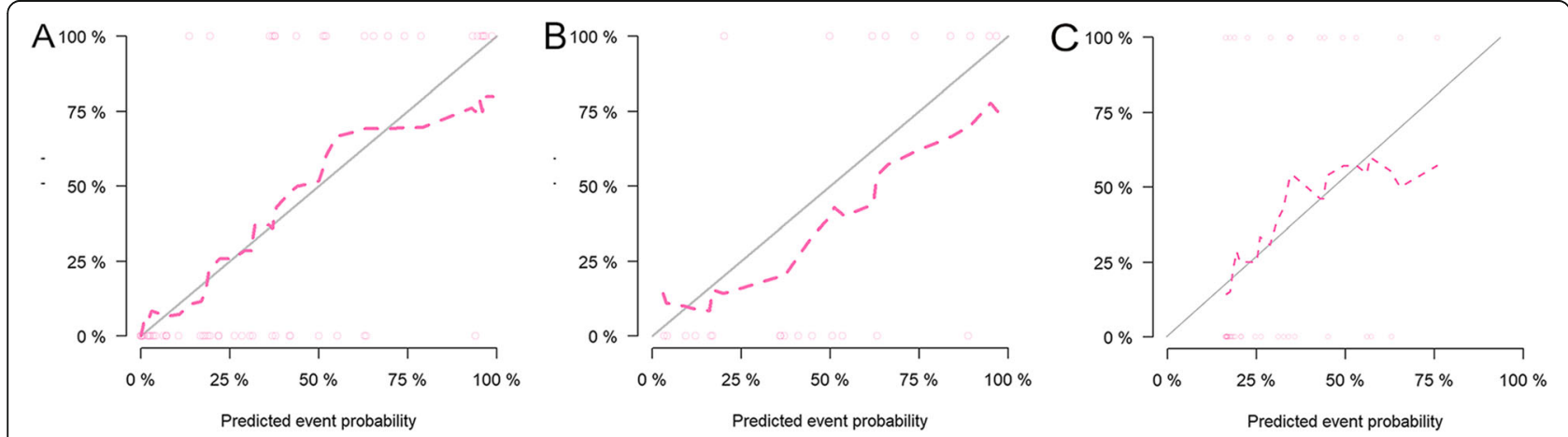

Fig. 6 Results of the Hosmer-Lemeshow test. The combined model fit well with the real situation both in the training cohort (a), internal and external validation cohorts $(\mathbf{b}, \mathbf{c})$ 


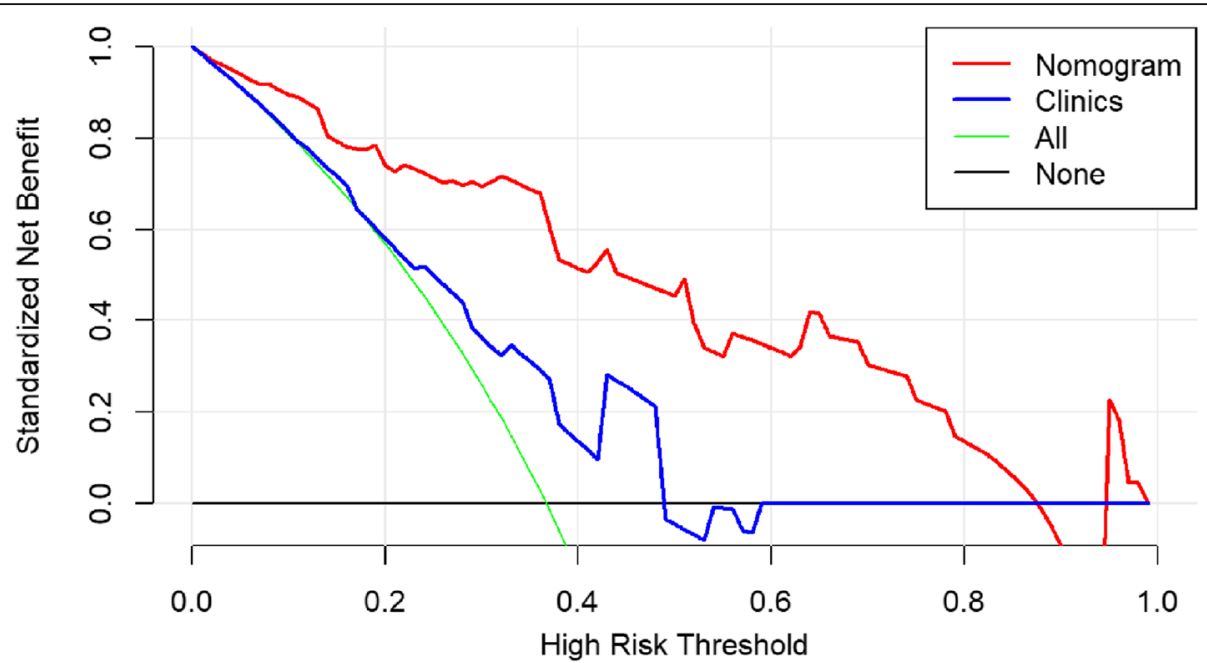

Fig. 7 Decision curve analysis demonstrated that the combined model had a higher net benefit than the traditional model at every threshold probability

MRI including T2-weighted images should be implemented into the predictive model to improve its robustness. Finally, we were not able to obtain CSF samples in the majority of their patients to identify M1 disease. But because of technical or sampling problems, CSF cytology may fail to detect the tumor cells. A more precise technique called circulating tumor DNA should be used in further study to improve the performance of the predictive model.

\section{Conclusions}

In summary, our preliminary study demonstrated that a preoperative-enhanced $\mathrm{T} 1$-weighted image-based radiomics could improve the prediction accuracy of CSF dissemination in children with MB. A nomogram constructed based on the radiomic signature and selected clinical features may provide more useful information for clinical decision making.

\section{Abbreviations}

MB: Medulloblastoma; CSF: Cerebral spinal fluid

\section{Supplementary Information}

The online version contains supplementary material available at https://doi. org/10.1186/s12957-021-02239-w.

\section{Additional file $1 .$.}

\section{Acknowledgements}

We thank all of the children included in this study for their participation.

\section{Declarations}

\section{Authors' contributions}

Dengbin Wang and Yuhua Li conceived the idea and designed the work. Chenqing Wu, Ting Gui, and Yuzhen Zhang obtained the data. Ming Liu checked the segmentation. Hui Zheng and Shao Feng Duan analyzed the data. Jinning Li and Huanhuan Liu interpreted the data. Hui Zheng wrote the manuscript. Jinning Li and Huanhuan Liu critically revised the manuscript. All authors are accountable for the contents of this work. The authors read and approved the final manuscript.

\section{Funding}

This study has received funding from the National Key Research and Development Program of China (No. 2017YFC0109003), Science and Technology Committee of Shanghai Municipality (No. 18411967500), and the Shanghai Municipal Health Commission (20204Y0164). The funders had no role in the study design, data collection and analysis, or preparation of the manuscript.

\section{Availability of data and materials}

The datasets generated and/or analyzed during the current study are not publicly available due to hospital policy but are available from the corresponding author on reasonable request.

\section{Ethics approval and consent to participate}

This study was approved by our Institutional Review Board, with reference XHEC-D-2020-136.

\section{Consent for publication \\ Not applicable.}

\section{Competing interests}

The authors declare that they have no competing interests.

\section{Author details}

'Department of Radiology, Xinhua Hospital affiliated to Shanghai Jiao Tong University School of Medicine, Shanghai, China. ${ }^{2}$ GE Healthcare, Pudong New Town, No.1, Huatuo Road, Shanghai 210000, China.

Received: 10 November 2020 Accepted: 12 April 2021

Published online: 22 April 2021

References

1. Ostrom QT, Gittleman H, Truitt G, Boscia A, Kruchko C, Barnholtz-Sloan JS. CBTRUS statistical report: primary brain and central nervous system tumors diagnosed in the United States in 2011-2015. Neuro-Oncology. 2018; 20(suppl 4):iv1-iv86.

2. Gajjar A, Chintagumpala M, Ashley D, Kellie S, Kun LE, Merchant TE, et al. Risk-adapted craniospinal radiotherapy followed by high-dose chemotherapy and stemcell rescue in children with newly diagnosed 
medulloblastoma (St Jude Medulloblastoma-96): long-term results from a prospective, multicentre trial. Lancet Oncol. 2006;7(10):813-20

3. Ellison DW, Kocak M, Dalton J, Megahed H, Lusher ME, Ryan SL, et al. Definition of disease-risk stratification groups in childhood medulloblastoma using combined clinical, pathologic, and molecular variables. J Clin Oncol. 2011:29(11):1400-7. https://doi.org/10.1200/JCO.2010.30.2810.

4. Louis DN, Perry A, Reifenberger G, von Deimling A, Figarella-Branger D, Cavenee WK, et al. The 2016 World Health Organization Classification of tumors of the central nervous system: a summary. Acta Neuropathol. 2016 131(6):803-20. https://doi.org/10.1007/s00401-016-1545-1.

5. Packer RJ, Siegel KR, Sutton LN, Litmann P, Bruce DA, Schut L, et al. Leptomeningeal dissemination of primary central nervous system tumors of childhood. Ann Neurol. 1985;18(2):217-21. https://doi.org/10.1002/ana.4101 80209.

6. Soares BP, Poretti A, Huisman TAGM. Magnetic resonance imaging to diagnose leptomeningeal spread of medulloblastoma in children: decreasing diagnostic uncertainty, moving in new directions. Pediatr Blood Cancer. 2017;64(8):e26514. https://doi.org/10.1002/pbc.26514.

7. Pang J, Banerjee A, Tihan T. The value of tandem CSF/MRI evaluation for predicting disseminated disease in childhood central nervous system neoplasms. J Neuro-Oncol. 2008:87(1):97-102.

8. Gillies RJ, Kinahan PE, Hricak H. Radiomics: images are more than pictures, they are data. Radiology. 2016;278(2):563-77. https://doi.org/10.1148/radiol.2 015151169.

9. Lambin P, Rios-Velazquez E, Leijenaar R, Carvalho S, van Stiphout RG, Granton P, et al. Radiomics: extracting more information from medical images using advanced feature analysis. Eur J Cancer. 2012;48(4):441-6. https://doi.org/10.1016/j.ejca.2011.11.036.

10. Kim Y, Cho HH, Kim ST, Park H, Nam D, Kong DS. Radiomics features to distinguish glioblastoma from primary central nervous system lymphoma on multi-parametric MRI. Neuroradiology. 2018;60(12):1297-305. https://doi. org/10.1007/s00234-018-2091-4

11. Huang Y, Liu Z, He L, Chen X, Pan D, Ma Z, et al. Radiomics signature: a potential biomarker for the prediction of disease-free survival in early-stage (I or II) non-small cell lung cancer. Radiology. 2016;281(3):947-57. https:// doi.org/10.1148/radiol.2016152234.

12. Liu $H$, Zhang $C$, Wang $L$, Luo $R$, Li J, Zheng $H$, et al. MRI radiomics analysis for predicting preoperative synchronous distant metastasis in patients with rectal cancer. Eur Radiol. 2019;29(8):4418-26. https://doi.org/10.1007/s00330018-5802-7.

13. Terterov S, Krieger MD, Bowen I, McComb JG. Evaluation of intracranial cerebrospinal fluid cytology in staging pediatric medulloblastomas, supratentorial primitive neuroectodermal tumors, and ependymomas. J Neurosurg Pediatr. 2010;6(2):131-6. https://doi.org/10.3171/2010.5. PEDS09333.

14. Miralbell R, Bieri S, Huguenin P, Feldges A, Morin AM, Garcia E, et al. Prognostic value of cerebrospinal fluid cytology in pediatric medulloblastoma. Swiss Pediatric Oncology Group. Ann Oncol. 1999;10(2): 239-41. https://doi.org/10.1023/a:1008363422189.

15. Huang YQ, Liang CH, He L, Tian J, Liang CS, Chen X, et al. Development and validation of a radiomics nomogram for preoperative prediction of lymph node metastasis in colorectal cancer. J Clin Oncol. 2016;34(18):2157-64.

16. Kim JY, Park JE, Jo Y, Shim WH, Nam SJ, Kim JH, et al. Incorporating diffusion- and perfusion-weighted MRI into a radiomics model improves diagnostic performance for pseudoprogression in glioblastoma patients. Neuro-Oncology. 2019;21(3):404-14. https://doi.org/10.1093/neuonc/noy133.

17. Su C, Jiang J, Zhang S, Shi J, Xu K, Shen N, et al. Radiomics based on multicontrast MRI can precisely differentiate among glioma subtypes and predict tumour-proliferative behaviour. Eur Radiol. 2019;29(4):1986-96. https://doi.org/10.1007/s00330-018-5704-8,

18. Yu J, Shi Z, Lian Y, Li Z, Liu T, Gao Y, et al. Noninvasive IDH1 mutation estimation based on a quantitative radiomics approach for grade II glioma. Eur Radiol. 2017;27(8):3509-22. https://doi.org/10.1007/s00330-016-4653-3.

19. Prasanna P, Patel J, Partovi S, Madabhushi A, Tiwari P. Radiomic features from the peritumoral brain parenchyma on treatment-naive multiparametric MR imaging predict long versus short-term survival in glioblastoma multiforme: preliminary findings. Eur Radiol. 2017;27(10):418897. https://doi.org/10.1007/s00330-016-4637-3.

20. Rodriguez Gutierrez D, Awwad A, Meijer L, Meijer L, Manita M, Jaspan T, et al. Metrics and textural features of MRI diffusion to improve classification of pediatric posterior fossa tumors. AJNR Am J Neuroradiol. 2014;35(5): 1009-15. https://doi.org/10.3174/ajnr.A3784.

21. Iv M, Zhou M, Shpanskaya K, Perreault S, Wang Z, Tranvinh E, et al. MR imaging-based radiomic signatures of distinct molecular subgroups of medulloblastoma. AJNR Am J Neuroradiol. 2019;40(1):154-61. https://doi. org/10.3174/ajnr.A5899.

22. Ramaswamy V, Remke M, Bouffet E, Bailey S, Clifford SC, Doz F, et al. Risk stratification of childhood medulloblastoma in the molecular era: the current consensus. Acta Neuropathol. 2016;131(6):821-31. https://doi.org/1 0.1007/s00401-016-1569-6.

23. Perreault S, Ramaswamy V, Achrol AS, Chao K, Liu TT, Shih D, et al. MRI surrogates for molecular subgroups of medulloblastoma. AJNR Am J Neuroradiol. 2014;35(7):1263-9. https://doi.org/10.3174/ajnr.A3990.

24. Kool M, Korshunov A, Remke M, Jones DT, Schlanstein M, Northcott PA, et al. Molecular subgroups of medulloblastoma: an international metaanalysis of transcriptome, genetic aberrations, and clinical data of WNT, SHH, Group 3, and Group 4 medulloblastomas. Acta Neuropathol. 2012; 123(4):473-84. https://doi.org/10.1007/s00401-012-0958-8.

25. Ding JL, Xing ZY, Jiang ZX, Chen J, Pan L, Qiu JG, et al. CT-based radiomic model predicts high grade of clear cell renal cell carcinoma. Eur J Radiol. 2018;103:51-6. https://doi.org/10.1016/j.ejrad.2018.04.013.

\section{Publisher's Note}

Springer Nature remains neutral with regard to jurisdictional claims in published maps and institutional affiliations.
Ready to submit your research? Choose BMC and benefit from:

- fast, convenient online submission

- thorough peer review by experienced researchers in your field

- rapid publication on acceptance

- support for research data, including large and complex data types

- gold Open Access which fosters wider collaboration and increased citations

- maximum visibility for your research: over $100 \mathrm{M}$ website views per year

At $\mathrm{BMC}$, research is always in progress.

Learn more biomedcentral.com/submissions 